\title{
When the disease is known but the patient is not. Outcome of 118 such patients at a Tertiary Care Centre
}

\author{
Gaurav Jaiswal, Praveen Kumar Tripathi, Vardan Kulshreshtha, \\ Tarun Kumar Gupta
}

Department of Neurosurgery, R.N.T Medical College, Udaipur, Rajasthan, INDIA

\begin{abstract}
This is a prospective study, carried out at the Department of Neurosurgery at M.B.G Hospital, R.N.T Medical College, Udaipur, Rajasthan (India). Many patients with history of trauma with head injury, whose identity cannot be ascertained, are admitted in our hospital. The management of these unattended patients from pre-hospital till discharge, rehabilitation or death is full of difficulties especially when surgical intervention has to be done. From March 16, 2015, until March 30, 2016, 118 consecutive patients unattended patients admitted in our department with history of head injury were enrolled in the study. Out of 118 patients, 107 (91\%) were male, most were in the age group of 30-39 years. In majority of patients, 115 (97\%) principal cause of head injury was road traffic accident. Majority of the patients $49(41 \%)$ had Glasgow coma scale $>13$ on admission. Twenty three patients 23 (20\%) died in hospital, 71 (60\%) patients had good recovery. During the course of treatment identity of 115 patients was established and $92(78 \%)$ patients, who survived were discharged to home. Three (3\%) patients were shifted to destitute home. All discharged patients were followed at 1 month and 6 month interval. $78(82 \%)$ \& $76(80 \%)$ patients showed good recovery (GOS) at 1 month \& 6 month respectively. One patient expired at home within one month of discharge and 6 patients lost to follow up at 6 months.
\end{abstract}

Key words: anonymous patient; head Injury; nameless patient; outcome; unknown patients; unidentified patient

\section{Introduction}

The total number of deaths in road accidents in India was 169107 during 2014 (1). The incidence of road traffic accident in Rajasthan in 2014 was 24639 and out of which the total number of deaths in road accidents in
Rajasthan was 10287 during 2014 (1), which was $6.08 \%$ of the total number of deaths in road accidents in India during the yearl. In India, the incidence of head injury is steadily increasing with urbanization and increasing number of vehicular population (2). Among 
the road traffic accidents $70 \%$ have head injury, among road accident deaths $70 \%$ are due to head injury. Majority of deaths occur during first 72 hours. Recently, number of fatal accidents has increased in India. Every minute there is an accident and every eight minute there is a death (3). Attempts to systematize care of patients with severe TBI have culminated in evidence-based guidelines issued by the joint task force between the Brain Trauma Foundation and the American Association of Neurological Surgeons $(4,5)$. Our institute which is a tertiary care centre of a developing country and a lot of severe head injury patients are being referred to us. Many such patients are often brought by policemen and bystanders and their identities are not known at the time of admission. These patients present a unique challenge in management. In this backdrop we prospectively studied demography, mode of injury, presentation, treatment, outcome and factors affecting outcome in this group of patients.

\section{Methodology}

This was a prospective study, conducted at Department of Neurosurgery, Maharana Bhupal Government Hospital, RNT Medical College, Udaipur, Rajasthan. From March 16, 2015, until March 30, 2016, 118 consecutive patients unattended patients admitted in our department with history of head injury were enrolled in the study. Note was made regarding demography, mode of injury, clinical presentation, and condition at admission, treatment given, hospital stay and outcome of the patients. All patients were clinically evaluated by a team comprising of doctors from surgical, medical and orthopaedics specialties in the emergency department and subsequently admitted and treated in neurosurgery unit. Plain CT scan head along with other imaging (if needed) like $\mathrm{X}$-rays, CT scan of spine, USG abdomen, MRI spine or contrast CT (abdomen chest) were carried out to rule out other injuries.

\section{Results}

Out of 118 patients, 107 (91\%) were male, and $11(09 \%)$ were female (Table 1). Only one patient was less than $20 \mathrm{yrs}$ of age, while the most patients 46 (39\%) were in the age group of 30-39 years. $12(10 \%)$ patients were $>60$ years of age group (Table 2). Road traffic accident was the major cause of injury in 115 (97\%) cases.

At the time of admission Glasgow Coma Scale (GCS) score of less than 8 was seen in 33 (28\%) cases. GCS of 8-12 was seen in 36 (31\%) cases. Plain CT scan of head revealed EDH in 12 (10\%) patients, 28 (24\%) had SDH, 20 (17\%) had cerebral contusions, 09 (08\%) had SAH, 04 (03\%) had diffuse axonal injury, 01 (01\%) patient had depressed fracture while in $44(37 \%)$ patients no abnormality was found on imaging (Table 3). Associated injuries were seen in total $64(54 \%)$ patients. 30 patients (25\%) had chest injury, 12 (10\%) had limb fractures, 20 (17\%) had maxillofacial injuries and only two had abdominal injuries.

Only 11 patients needed neurosurgical intervention where decompressive craniectomy was carried out in 6 (5\%), craniotomy was in 4 patients (3\%), 1 patient (1\%) underwent depressed facture elevation 
(Table 4). During treatment, 20 patients (17\%) had chest complications, $9(8 \%)$ had wound infection, 4 patients developed meningitis (Table 5). At the time of discharge, 81 patients (68\%) had GCS of 13-15 and eight (07\%) had GCS score 12-8, only 4 patients had GCS less than 8 at time of discharge. According to Glasgow outcome scale (GOS) of these patients, $71(60 \%)$ had good recovery, 11 (09\%) had moderate disability, 8 (07\%) had severe disability, 05 (04\%) remained vegetative and $23(20 \%)$ died during treatment. During the course of treatment identity of $110(93 \%)$ patients could be identified and 92 (78\%) patients who survived were sent home with relatives, 3 (03\%) patients who remained unidentified were sent to destitute home. All patients who were discharged followed at 1 month and 6 month intervals. 78 (82\%) \& 76 (81\%) patients showed good recovery (GOS) at 1 month \& 6 month respectively, 1 patient died at home. 6 of the discharged patients lost to follow up at 6 months.

TABLE 1

Demography (gender wise) of head injury in unknown patients admitted in neurosurgery: $(\mathrm{N}=118)$

\begin{tabular}{|l|l|l|}
\hline Demography (Gender) & No. of patients(n-118) & Percentage \\
\hline Male & 107 & 91 \\
Female & 11 & 9 \\
\hline Total & 118 & 100 \\
\hline
\end{tabular}

TABLE 2

Demography of head injury in unknown patients admitted in neurosurgery: $(\mathrm{N}=118)$

\begin{tabular}{|l|l|l|}
\hline Demography & No. of patients(n-118) & Percentage \\
\hline Mean age(years) & & \\
\hline Age group & & \\
\hline$<20$ & 1 & 1 \\
\hline $20-29$ & 28 & 23 \\
\hline $30-39$ & 46 & 39 \\
\hline $40-49$ & 22 & 19 \\
\hline $50-59$ & 9 & 8 \\
\hline$>60$ & 12 & 10 \\
\hline Total & 118 & 100 \\
\hline
\end{tabular}


508 | Jaiswal et al - When the disease is known but the patient is not

TABLE 3

Clinical characteristic and type of lesions in head injury of unknown patients: $(\mathrm{N}=118)$

\begin{tabular}{|l|l|l|}
\hline Cause of head injury & Number of patients (118) & Percentage \\
\hline Road traffic accident & 115 & 97 \\
Other (FALL from train) & 3 & 3 \\
\hline Glasgow coma scale at admission & & \\
$13-15$ & 49 & 41 \\
$12-8$ & 36 & 31 \\
$<8$ & 33 & 28 \\
\hline Type of brain injury (major component) & & \\
Extradural haematoma (EDH) & 12 & 10 \\
Subdural haematoma(SDH) & 28 & 24 \\
Cerebral contusion & 20 & 17 \\
Depressed fracture & 01 & 01 \\
Diffuse axonal injury(DAI) & 04 & 03 \\
Subarachnoid haemorrhage(SAH) & 09 & 08 \\
No observable abnormality on imaging & 44 & 37 \\
\hline Other injuries & & \\
- Chest injury & 30 & 25 \\
- Abdominal injury & 02 & 02 \\
- Limb fracture & 12 & 10 \\
- Maxillofacial & 20 & 17 \\
\hline Other emergency surgical speciality & ICD insertion in 20 patients & 17 \\
procedure & & \\
\hline
\end{tabular}

TABLE 4

Surgical Procedures Performed $(\mathrm{N}=11)$

\begin{tabular}{|l|l|l|}
\hline Types of treatment/ Surgery & No. of patients (11 of 118) & Percentage \\
\hline Decompressive Craniectomy & 6 & 05 \\
Craniotomy & 4 & 03 \\
Depressed fracture elevation & 1 & 01 \\
Burr hole evacuation & - & - \\
\hline Total & 11 & 09 \\
\hline
\end{tabular}


TABLE 5

Complications during treatment of the patients (N-118)

\begin{tabular}{|l|l|l|}
\hline Complications & No. of patients & Percentage \\
\hline - Pneumonia & 20 & 17 \\
- Meningitis & 04 & 03 \\
- Septicemia & - & - \\
- Wound infection & 09 & 08 \\
- CSF leak & - & - \\
- Hydrocephalus & - & - \\
\hline Total & 33 & 28 \\
\hline
\end{tabular}

TABLE 6

Outcome and destination of patients during discharge $(\mathrm{N}=118)$

\begin{tabular}{|l|l|l|}
\hline Outcome & No. of patients (118) & Percentage \\
\hline Glasgow outcome scale & & \\
Good recovery & 71 & 60 \\
Moderate disability & 11 & 09 \\
Severe disability & 08 & 07 \\
Vegetative state & 05 & 04 \\
Death & 23 & 20 \\
\hline Glasgow Coma scale at discharge & & \\
$13-15$ & 81 & 68 \\
$8-12$ & 08 & 07 \\
$<8$ & 04 & 03 \\
\hline Discharged location & & 78 \\
Home & 92 & 03 \\
Destitute Home & 03 & \\
\hline
\end{tabular}

TABLE 7

Outcome during follow up of patients after discharge (N-95)

\begin{tabular}{|l|l|l|}
\hline Outcome (Glasgow outcome scale) & After 1 month & After $\mathbf{6}$ month \\
& $\mathbf{N = 9 5}$ & $\mathbf{N = 9 4}$ \\
\hline Good recovery & 78 & 76 \\
Moderate disability & 05 & 04 \\
Severe disability & 07 & 04 \\
Vegetative state & 04 & 04 \\
Death & 01 & 00 \\
\hline Lost to follow & 0 & 06 \\
\hline
\end{tabular}




\section{Discussion}

In a study by Ahmad et al (6), out of 325 unidentified patients, there were 9 (3\%) patients in the paediatric age group and 16 (5\%) patients were above 60 years of age. Of these, 193 (65\%) could be identified during the hospital stay. An additional 40 (13\%) patients were sent home after they regained memory of their addresses. Forty seven patients (15\%) died without their identities being established. Seventeen (6\%) patients remained unknown and were sent to rehabilitation/poor homes with the help medical social worker: All pediatric patients were identified (6).

In study of Wanger et al, they reported approximately one third of patients with moderate head injury and half of patients with severe head injury were operated, most of them being for cerebral contusions and/or subdural hematomas7. Mortality following head injury has been reported to be in the range of $39-51 \%(8,9)$.

Study by Liew BS et al. showed both known and unknown head injury patients, among 72 patients of head injury eleven patients (15\%) died during hospitalization. There were only sixty one ( $85 \%)$ patients were discharged from hospital, where by twenty nine (40\%) with good outcome (GOS 4 and 5) while the remaining thirty two (44\%) patients were with either severe disability or vegetative state. Only one patient continued to suffer severe disability, while the rest had moderate or good recovery (10). In another study there were twelve patients (17\%) under the age of twenty years, only 5 patients (7\%) were above 60 years. Twenty patients $(29 \%)$ were treated by surgery, most often for cerebral contusion (33 patients, 47\%) (11).

Till date no prospective study has been conducted to assess outcome of such unidentified patients who underwent neurosurgical procedures. In our study 118 patients were treated of which 11 patients needed surgical intervention where decompressive craniectomy was carried out in $6(54 \%)$, craniotomy was in $4(37 \%)$, One patient (9\%) underwent depressed fracture elevation. Remaining107 (91\%) patients were treated conservatively. Among these patients, 71 patients (60\%) had good recovery, 11 patients $(09 \%)$ had moderate disability, 8 patients (07\%) had severe disability, 5 patients (04\%) remained vegetative and 23 patients (20\%) died during treatment. During the course of treatment identity of $110(93 \%)$ patients could be identified and 92 (78\%) patients who survived were sent home with relatives, $3(03 \%)$ patients who remained unidentified were sent to destitute home.

These unidentified patients with unknown identities present considerable challenges in their management. They are usually found lying on road in unconscious state and brought to hospital by policemen or bypassers who are ill equipped and often ignorant in handling patients with severe injuries. Their prehospital management is usually improper and lack of proper transfer facilities, in ambulances, further aggravates their condition. We receive many such patients from peripheral hospitals, because of lack of proper facilities there. Very often such patients are destitute and their injuries are compounded by presence of debility because 
of poor nutrition, other medical conditions like diabetes, hypertension, substance abuse and mental illnesses. Therefore, it is imperative that these patients be evaluated with a very high index of suspicion for above conditions. During their hospital stay, the role of paramedical staff is of paramount importance; their daily nursing care in absence of a relative is a challenging task. It needs a team of trained and empathetic nursing staff along with a physiotherapist, dietician, psychologist, and social worker who can help and rehabilitate them. Performing neurosurgical procedures in such patients is not devoid of difficulties ranging from consent to rehabilitation. Existing infrastructure and much trained staff, enthusiastic multidisciplinary team, social workers and media personnel help many unidentified patients with severe head injury in rehabilitation. It is highly gratifying for the treating team to see such patients return back to our outpatient department with their relatives. We believe that there is an urgent need to sensitize the general public and police about the transportation and prehospital management of such severe head injury patients. Our peripheral hospitals need to be well equipped for treatment of such patients. Treatment of such unknown patients can entail a huge expenditure and therefore, every hospital should allocate funds for the above purpose and only those patients who are in need of higher medical care should be referred to higher centre. The role of social media and general media also has a capacitive role in spreading awareness and rehabilitating unidentified patients.

\section{Conclusion}

Management of unidentified head injury patients are posed with challenges. Like other patients initial management is of vital importance. Outcome of these patients is comparable to patients who are accompanied by their relatives. Their management from prehospital to treatment and discharge from hospital is quite challenging especially, when patient requires operative interventions, in ICU and meeting nutritional standards. There mean hospital stay and ICU stay is sometimes longer in comparison to similar patients as they may remain unidentified. They need special care for which staff should be well trained and hospital must have economic resources. Hospital should have a protocol system to deal with such patients especially when they require neurosurgical interventions. A good network of social workers and active involvement of media helps in identification and rehabilitation of these patients.

\section{Correspondence}

Dr. Gaurav Jaiswal, Associate Professor, Department of Neurosurgery, RNT Medical College, Udaipur (Rajasthan)

E-mail:drgauravjaiswal@gmail.com

\section{References}

1.http://ncrb.gov.in/StatPublications/ADSI/ADSI2014/c hapter-1A\%20traffic\%20accidents.pdf

2.Report of road safety cell: Ministry of Transport Govt of India, January 1993.

3.Head injuries: A neglected field in India. National Medical Journal 1991; 4:53-44.

4.Guidelines for the management of severe head injury. The Brain Trauma Foundation. The American Association of Neurological Surgeons, The Joint Section 
512 Jaiswal et al - When the disease is known but the patient is not

on Neurotrauma and Critical Care. J Neurotrauma. 1996;11:641-734.

5.The Brain Trauma Foundation. The American Association of Neurosurgical Surgeons. The Joint Section on Neurotraumaand Critical Care. Trauma systems. J Neurotrauma. 2000;17:457-627.

6.Ahmad FU, Mahapatra AK, Mehta VS. Outcome of unknown head injury patients at a tertiary care neurosurgical centre: Neurology India 2006; 54:73-4.

7.Wanger AK, Sessere HC, Hammond FM, et al. International Traumatic Brain Injury: Epidemiology and risk factors associated with Severity and Mortality. J Trauma 2000; 49:404-10.

8.Lannoo E, Van Rietvelde F, Colardyn F, et al. early predictors of mortality and morbidity after severe closed head injury. J Neurotrauma 2000;17:403-14.
9.Bulger Em, Nathens AB, Rivara FP, et al. Brain Trauma Foundation: Management of severe head injury: Institutional variations in care and effect on outcome. Crit Care Med 2002; 30:1870-6.

10.Liew BS, Johari SA, Nasser AW, Abdullah J. Severe Traumatic Brain Injury: Outcome in Patients with Diffuse Axonal Injury Managed Conservatively in Hospital Sultanah Aminah, Johor Bahru- An Observational Study. Med J Malaysia 2009; 64: 280-8.

11.Outcome of head injury in unknown patients at Level1 apex trauma centre Nath, Haradhan Deb et al. The Indian Journal of Neurotrauma, Volume 8, Issue 1, 11 15. 